\title{
Analysis of three dimensional torso shape and bodice pattern shape of young Japanese Women
}

\author{
Keiko WATANABE* \\ Kyoto Women's University, Kyoto, Japan
}

\begin{abstract}
The purpose of this study was to clarify factors that reflect the diversity of the shape of the body of young Japanese women. One hundred fifty-six young, healthy Japanese women were measured using VIVID 910 non-contact 3D digitizers and the data for each individual were transformed to a homologous model to enable statistical analysis. These models were then analyzed with principal component analysis (DHRC-HBS-PCA). The three-dimensional body surface of each subject was developed by LookStailorX. The relationships between the principal components and shape of the developed surface were observed in the clothing alteration. These factors could be effective input variables for pattern alteration in addition to ordinary size variables. The application of "body type grade" was examined.
\end{abstract}

Keywords: mass-customized clothing, pattern alteration, torso, surface development

\section{Introduction}

Female customers report fit as a major reason for not purchasing ready-to-wear clothes. We can observe many fitting problems even for average-sized young women. Although the percentage of ready-made clothing among all clothing is very high, Matsuyama reported that $81 \%$ of consumers replied that ready-to-wear clothes do not fit their body, and $75 \%$ of consumers and apparel manufacturers were interested in mass-customized clothing in Japan [1]. Lee [2] also indicated that fit to body shape was the most important feature females wanted to customize (88\%) in the US. Much attention has been given to mass-customized clothing. The Ministry of Economy, Trade and Industry of Japan discussed the practical application of "on-demand fashion". Mass customization or "on-demand fashion" might be explained as providing customers with exactly what they want and when they want it, at a reasonable price. Made-to-measure clothing businesses called "size order", in which sizes such as sleeve length and waist girth are adjusted, are seen. On the other hand, measurements such as length and girth are calculated from three-dimensional body scanning data. The measurements are then used to develop customized patterns to fit individual customers [3]. However, these methods cannot always accommodate the shape of the pattern to the customer's body shape. Consumers seek more personalized pattern alterations without a trial fitting for tight-fitting clothes such as a suit or wedding dress.

To improve apparel fit, technology that can automatically accommodate the pattern shape to the customer's body shape is necessary. "Body type grading" using an apparel CAD system is one possibility. In the case of size grade in the apparel CAD system, the apexes or specific point of measurement for each pattern piece is defined as grade points. Grade rules are applied to the grade point, which will increase or decrease the measurement of a specific pattern piece at a specified point. Grade rules determine which grade points are moved, and how much the points are moved on the $x-y$ axis. If the grading rule of "Body type grade is established, the same CAD system could be used for this purpose. Although there is traditional or empirical knowledge of pattern alteration to getting a well-fit pattern, the clothes need basting or re-basting. In order to establish a "Body type grade rule", it is important to clarify the mathematical relationship between flat patterns and actual body shape.

The first objective of this study was to clarify the principal components of the torso shape, which show what parts of the torso are most varying among young Japanese women. Then, the relationships between the principal components and the shape of the body surface of the subjects were observed in terms of clothing alteration or "Body type grade".

\footnotetext{
*watanaba@kyoto-wu.ac.jp; +81- 75-531-7162; http://www.kyoto-wu.ac.jp/index-e.html
} 


\section{Methods}

\subsection{Subjects and measurement}

The subjects were 156 healthy Japanese women aged between 18 and 25 years. They were asked to put on a sports bra because we intended to design outerwear and a close-fitting elastic camisole on it. Eighteen landmarks were pasted on the body surface. The base lines, such as neck base line, armhole line and shoulder seam line, were drawn on the skin with eyeliner and the waist line was marked with $3 \mathrm{~mm}$ wide tape.

The subject was asked to maintain the natural standing position, with their feet $20 \mathrm{~cm}$ apart from each other and their arms abducing approximately 20 degrees. After they stood in the correct position, the tips of two horizontal bars were adjusted to touch the subject's back in the occipital region and sacral region, respectively. The subject was asked to maintain contact with the tips of the bars to avoid a swaying motion during the measurement. VIVID 910 non-contact 3D digitizers (Konica Minolta Sensing, Inc., Tokyo, Japan) were used in this study. The height and angle of the VIVID digitizers could be freely moved, and the digitizers were arranged in such a way that there was no loss of data at the side of the torso and the shoulders. The data from the six digitizers were consolidated using software that we had designed. The origin was unified based on the landmarks; the front neck point was the origin of the $\mathrm{X}$-axis (transversal direction), the trochanter point was the origin of the $\mathrm{Y}$-axis (vertical direction) and the right side neck point was the origin of the Z-axis (sagittal direction). The models were not rotated around any axis. The areas outside of the armhole line, above the neckline and below the hipline were cut off.

\subsection{Analysis of 3D body shape}

The torsos were expressed by more than 180,000 points. To enable statistical analysis, the data for an individual were transformed to a homologous model (Fig.1) by the HBM (Homologous Body Modeling) software (Digital Human Research Center, Tokyo, Japan) [4]. Homologous modeling is a reconstruction of data points in such a way that the data for each subject will consist of the same number of data points of the same topology, and each data point will have the same anatomical position across subjects. Homologous models were not rotated at all to make them uniform, because gravity, which garments hang down parallel to, is very important in clothing design. Gravity causes the center and side seam to hang perpendicular to the floor. These models were then analyzed with principal component analysis using HBS-PCA (Human Body Statistica, Digital Human Research Center, Tokyo, Japan) [5] to clarify which body parts showed major differences among the individuals.
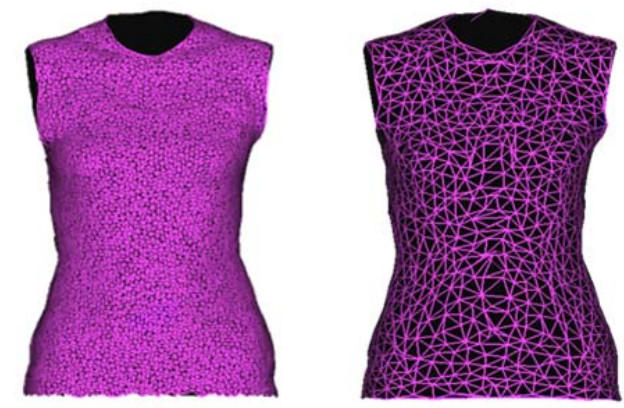

Fig. 1. The polygon data of a subject after cutting off the unnecessary area for analysis (left) and Homologous model of the same subject (right).
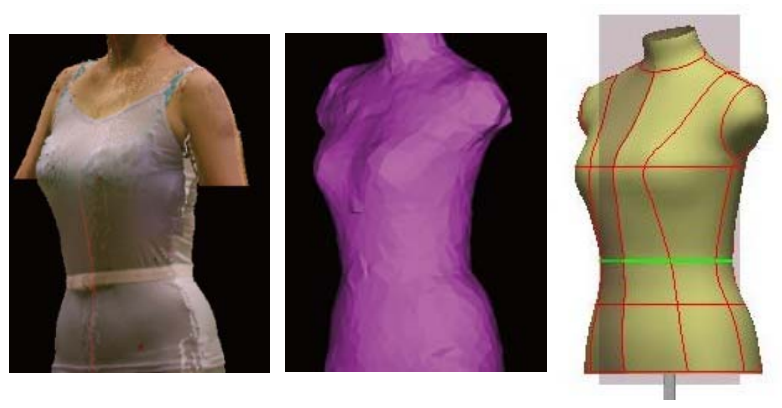

Fig. 2. The procedure of getting a subject's three-dimensional measured data as the digital virtual dress dummy of "lookStailor X".

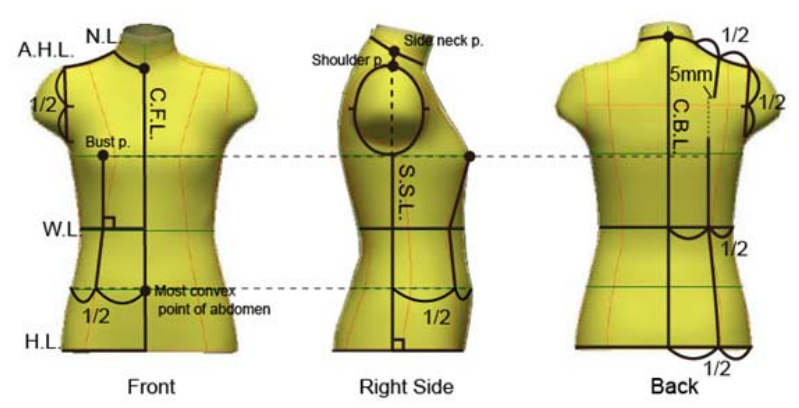

Fig. 3. The base lines and darts used as cutting line to develop the surface of the torso.

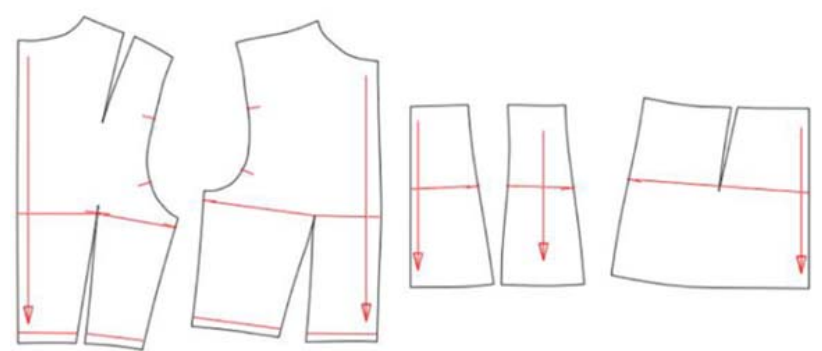

Fig. 4. A set of developed surfaces of the torso and the grain of each piece. 


\subsection{Body surface development}

We tried to observe how the principal components showing variation of the torso among individuals made a difference in the flat pattern shape. The body surface of each subject, which will be the basis of the clothing pattern, was calculated by 3D CAD software named "LookStailorX" (Desital fashion Co., Osaka, Japan). The procedure was as follows: the number of data points for each three-dimensionally measured data was redacted to approximately 1500 points, converted to OBJ data format, and entered into the 3D CAD software as a digital virtual dress dummy (Fig. 2). A tight fitting cloth was automatically generated on it. Then, the neck base line, armhole line, front median line, back median line, waist line, hip line, shoulder seam line and side seam line, the last of which is defined as the perpendicular line from the shoulder point, were drawn on it as a cutting line. Darts were defined referring to Bunka's basic pattern and drawn as shown in Fig. 3. The pieces inside of these lines were cut out and transformed into a surface with 2D coordinates (Fig. 4). The distortions of the developed
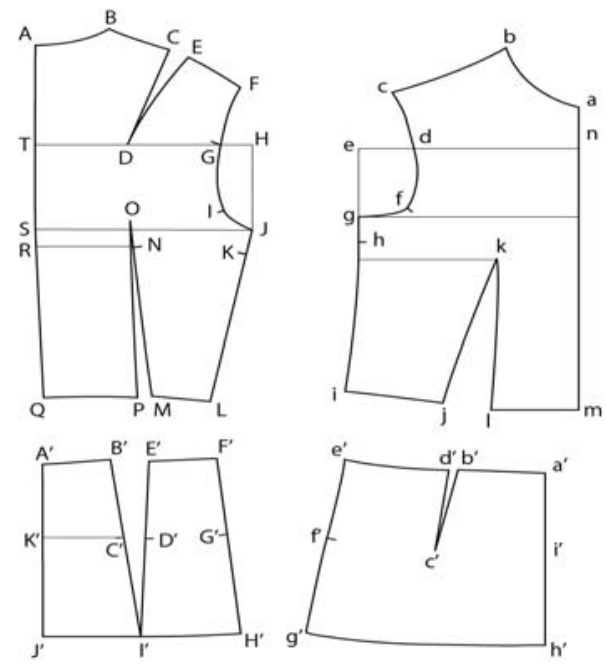

Fig.5. Fifty-four $x$-y coordinate values of the apexes of each surface and landmarks.

surfaces were within $2 \%$.

The surface data were entered into the apparel CAD system named CREACOMPO (Toray Advanced Computer Solution, Inc., Tokyo, Japan). Fifty-four $x-y$ coordinate values of the apexes of each surface and the landmarks, such as the across chest point (d), across back point (G), bust line on the side seam, back darts and back median line ( $h, K, N, R)$, which might be "grading points" of the pattern, were calculated (Fig. 5). The origins were the back neck point for the upper back piece, front neck point for the upper front surface, back waist point for the lower back surface, and front waist point for the lower front surface.

\subsection{Approaches of "Body type grading" and the trial fitting}

It is thought that some factors extracted in this study will be useful for personalized pattern alterations. The seventh principal component was the focus in an approaches of body type grading. The subjects were divided into three groups by the score of the principal component. The simplified shapes of the surfaces were drawn using 54 average $x-y$ coordinates calculated for each group. The differences in the $x-y$ coordinates among the 3 groups were examined from the viewpoint of clothing design. The specific points were selected and the values of the difference in $x-y$ coordinates were used for the tentative grade rule of "Body type grading". A jacket's sloper (basic pattern) in a 9AR (height $=158 \mathrm{~cm}$, bust girth $=83 \mathrm{~cm}$, waist girth $=64 \mathrm{~cm}$, hip girth $=91 \mathrm{~cm}$ ) was drawn by Ono's method [6] and points on the sloper were moved according to the rules. The jackets were sewn with wool textile. Two subjects, whose body size and shape were similar to each other, were selected from the High Score group and Low Score group, respectively, as trial fitting models. They tried on two types of the jackets, pictures were taken from 4 directions, and the fit was evaluated.

\section{Results and discussion}

\subsection{Principal component analysis of 3D body shape}

Wide variations in the shape of the torsos were seen even though our subjects were young university students of Japanese ethnicity. The homologous models were analyzed by principal component analysis. Ten principal components (PCs) were extracted and the principal component scores for each individual were calculated. For each principal component, the subjects with a low principal component score and those with a high principal component score were compared. Furthermore, average +1S.D. models and average -1S.D. models for each principal component were generated by HBS and they were compared (Fig. 6). As a result, the principal components were interpreted as follows. 

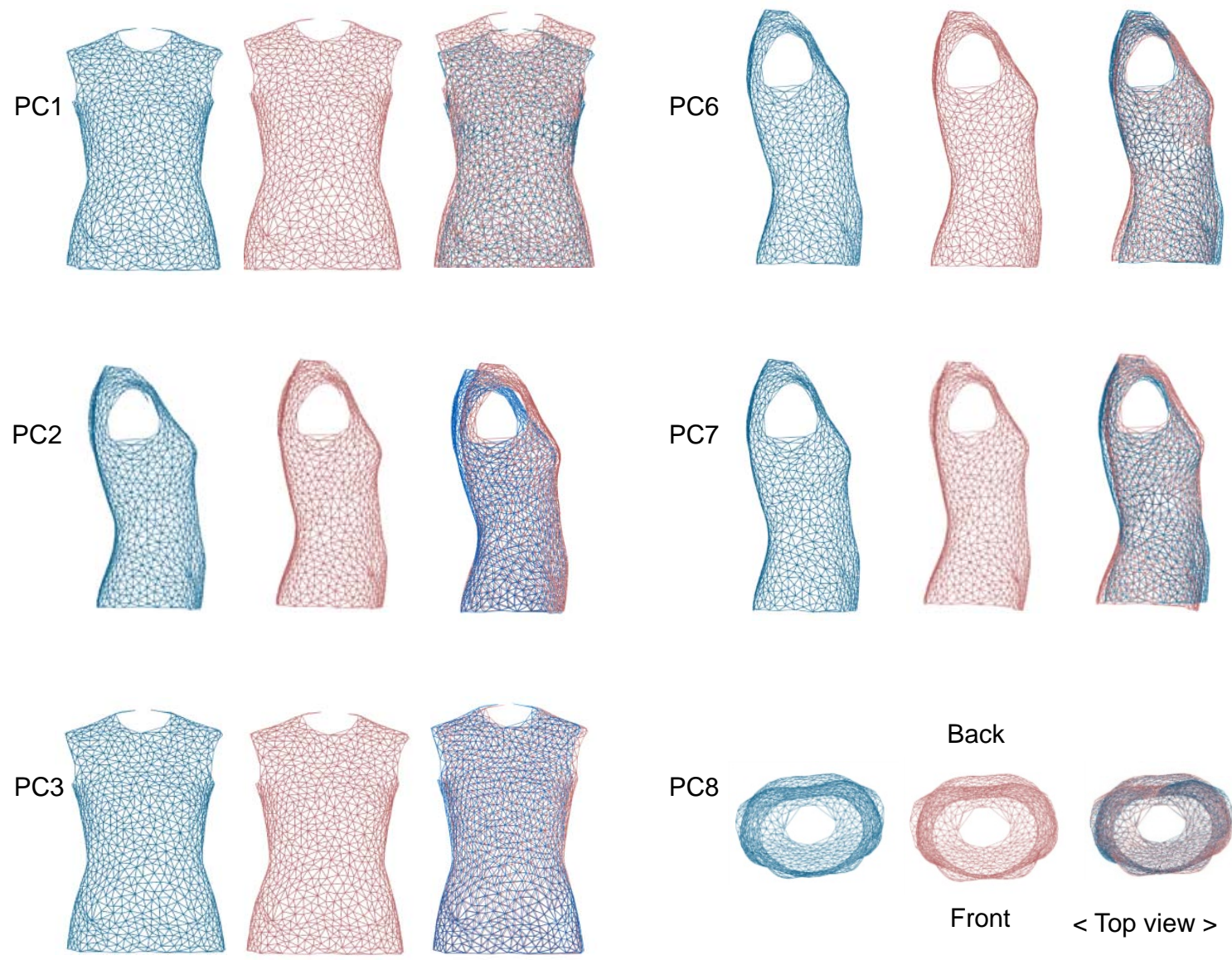

Back
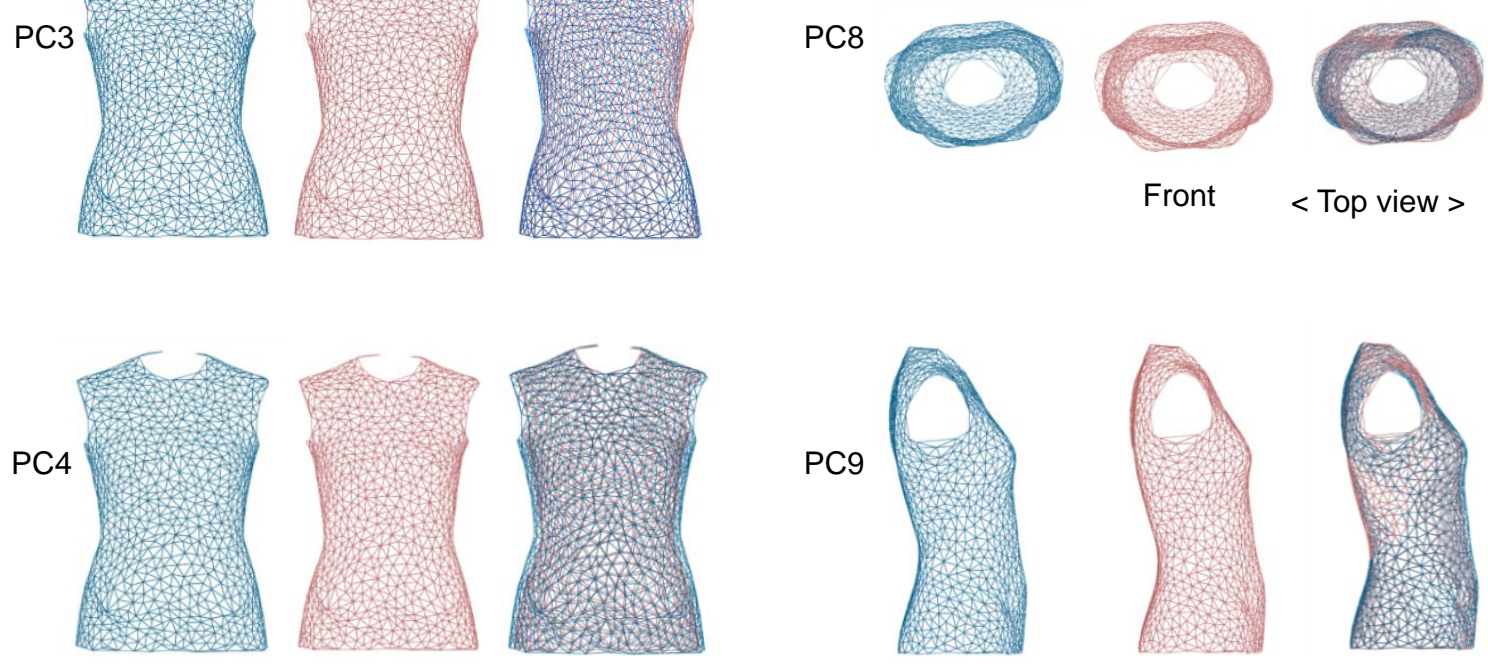

PC5
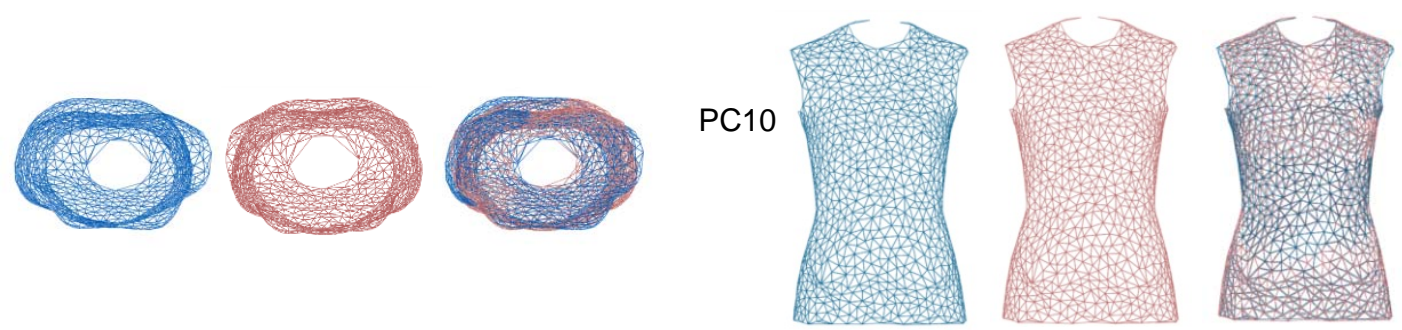

$-1 S . D$.

+1S.D.

superimposed

-1S.D.

+1S.D.

superimposed

Fig.6. The modes showing average -1S.D. and +1S.D. for each principal component, which is generated by HBS. 
PC1 was the height of the torso from the hip line to the cervical point. PC2 was inclination of the torso toward or backward above the waistline. PC3 was inclination of the torso to the right or left side. PC4 was the girth of the whole torso. PC5 was distortion and inclination of the shoulder relative to the pelvis area. The right shoulder point was located more foreword and the left shoulder point was located more backward for the lower scored subjects and the location of the shoulder point was opposite for the higher scored subjects, although the pelvis area was symmetrical. PC6 was the S-shape of the back showing concave lumbar area and protrusion of the buttock area. PC7 was the factor showing curved or straightened (erect) back. For PC8, the torso was rotated to the right or left side, but the line connecting both shoulder points and the line connecting both trochanter points were almost parallel. When the subjects were measured, it was checked if they put their toes on the line parallel to the $X$-axis and their feet parallel to the Z-axis. Then, PC8 was considered as distortion of the whole torso relative to the feet. PC9 was protrusion of the bust. PC10 was the width of the shoulder area.

The extracted factors can be roughly classified into four categories: size like height (PC1) or girth (PC4), inclination of the torso to the sagittal (PC2) or transversal (PC3) direction, protrusion of lower torso (PC6), back (PC7), bust (PC9), or shoulder (PC10) area and distortion of trunk of the body (PC5) or inferior limb girdle (PC8). As the principal component analysis used the 3D coordinate value of each apex of polygon, and as the correlation coefficients between the coordinate values located nearby had a tendency to be higher, these results could be said to be natural or appropriate.

On the other hand, these factors are also valuable from the viewpoint of clothing design. It is well known that the height of the torso (PC1) is highly correlated with the height of the individual, and the girth of the torso (PC4) is correlated with bust girth. In size standard such as JIS or ISO, height and bust girth are one of the control dimensions. Shoulder width (PC10) is not a control dimension; it is well known that it does not show high
Table 1. Body dimensions of the Low Score, Middle Score and High Score groups.

\begin{tabular}{|c|c|c|c|c|c|c|}
\hline \multirow{2}{*}{ Item } & \multicolumn{2}{|c|}{ Lower $(\mathrm{N}=30)$} & \multicolumn{2}{|c|}{ Midle $(\mathrm{N}=103)$} & \multicolumn{2}{|c|}{ Higher $(\mathrm{N}=23)$} \\
\hline & Mean & S.D. & Mean & S.D. & Mean & S.D. \\
\hline & $\mathrm{cm}$ & $\mathrm{cm}$ & $\mathrm{cm}$ & $\mathrm{cm}$ & $\mathrm{cm}$ & $\mathrm{cm}$ \\
\hline Bust girth & 82.0 & 5.5 & 80.6 & 5.3 & 80.6 & 3.9 \\
\hline Waist girth & 66.9 & 4.4 & 65.5 & 5.2 & 65.3 & 3.5 \\
\hline Hip girth & 91.0 & 4.7 & 91.1 & 4.5 & 91.9 & 3.8 \\
\hline Waist length & 38.0 & 1.9 & 37.6 & 1.7 & 37.8 & 1.7 \\
\hline Across shoulder & 37.4 & 2.0 & 37.4 & 1.7 & 37.5 & 1.6 \\
\hline & degree & degree & degree & degree & degree & degree \\
\hline Shoulder slope & 22.1 & 4.2 & 21.5 & 4.4 & 22.6 & 4.0 \\
\hline
\end{tabular}

The subjects were divided into 3 groups according to their PC7 score:

Low Score (under -10) group, Middle Score group, and High Score $($ ver +10$)$ group.

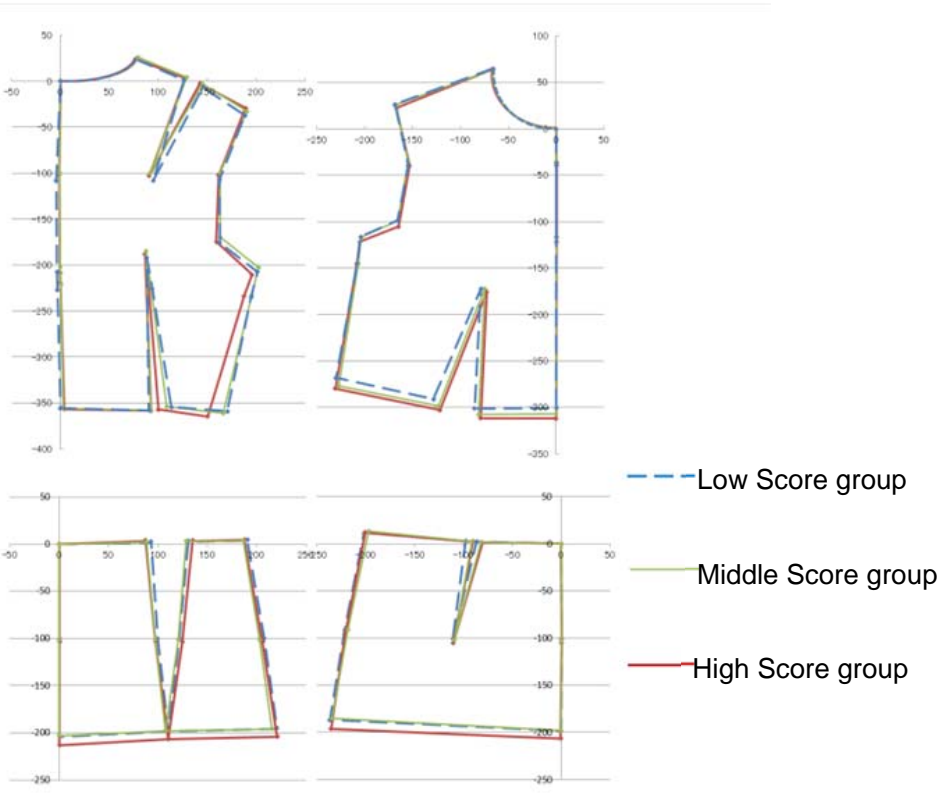

Fig. 7. Simplified shapes of the body surface drawn using the average value of the points on them in each group.

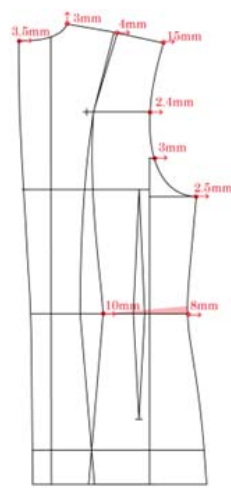

Low Score group

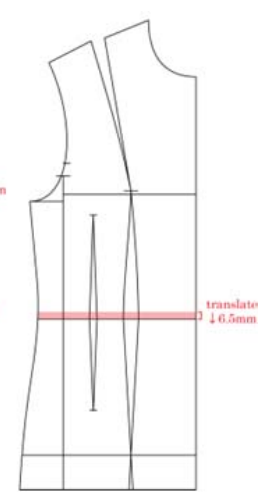

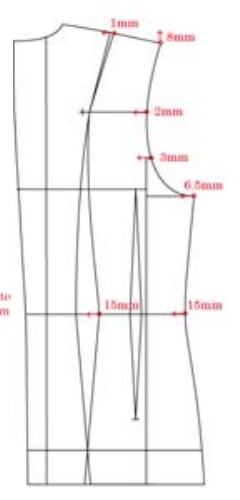

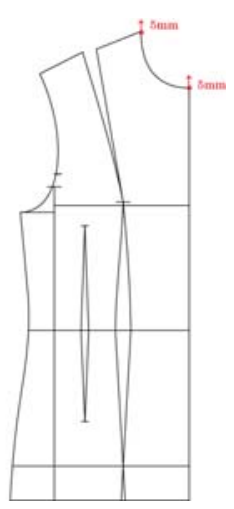

High Score group
Fig. 8. The major grade points and the distance and direction in which the points were moved. 
correlations with height or bust girth. There are fitting problems related to shoulder width, when ready-made clothes were selected using the size standard. These results are in agreement with studies of body size data measured by the traditional method using tape measure and anthropometer. Shoulder width might be altered with the dimension when "on-demand fashion" is delivered. The factors of inclination of the torso to the sagittal (PC2) or transversal (PC3) direction and the factors of $\mathrm{S}$-shape of the lower torso (PC6) or back (PC7) are related to one's spine or posture. Protrusion of the bust (PC9) varied widely and sometime caused serious fitting problems. These shapes have been considered to be the key points in clothing alteration; then, these factors could be assumed to be an important factor for Body type grading. Even with the distortions, when we design clothes, we cannot ignore this kind of asymmetry of the body and need to carefully consider which side or which part of the body shape or size should be used.

In my previous study, 106 of these models were then analyzed with the Multi-Dimensional Scaling method using DHRC-HBS-MDS and 5 factors were extracted as a result [7]. The first factor can be interpreted as the height above the hip line to the cervical point. The second factor can be interpreted as the depth of the lumbar spine area. The third factor was interpreted as girth. The fourth factor was interpreted as right or left distortion of posture. The fifth factor was interpreted as inclination of the torso forward or backward. Although similar factors were extracted from both analyses, the principal component analysis resulted in a greater number of factors. Principal component analysis is more effective for clothing alteration because the shape of an area of the body is important and it is insufficient to only consider factors of such rough shape when aiming at a certain level of fit.

\subsection{Body surface development and pattern alteration}

Next, it was tested whether the extracted factors could be used for body type grading. The subjects were divided into three groups according to their PC6, PC7 and PC9 scores. Then, the average coordinate values of 54 points on the surface were calculated for each group and compared with each other. Differences were seen and interpreted as reflecting the feature of the body shape from the viewpoint of clothing construction or alteration.

Therefore, we tried to use PC7 to alter the jacket sloper. The subjects were divided into the Low Score (under -10) group $(n=23,14.7 \%)$, the Middle group $(n=103,66.0 \%)$ and the High Score $($ over +10$)$ group ( $n=30,19.2 \%)$ by the PC7 score. The major body dimensions were not significantly different among the three groups (Table 1), and corresponded to "9AR" of JIS size standard. Simplified shapes of the body surface were drawn using the average value of the points for each group (Fig. 7). Differences were seen in the angles of the back shoulder darts, and back waist darts of the upper and lower torso. The shoulder point, armhole line and side seam line of the upper back were different among the three groups. Furthermore, the front length of the upper torso was shorter in the Low score group, whose back was more curved.

Based on these results, a tentative grade rule was defined and applied to the jacket sloper. The major grade points and the moved distance and direction of the points are shown in Fig. 8. The other points were proportionally moved. A standard jacket sloper and two altered slopers were sewn and tried on by Subjects $A$ and B, whose body dimensions were similar to each other and corresponded to "9AR" of JIS size standard. Subject A was selected from the Low Score group and her principal component score of PC7 was -14.1. Subject B was selected from the High Score group and her principal component score of PC7 was +12.6 .
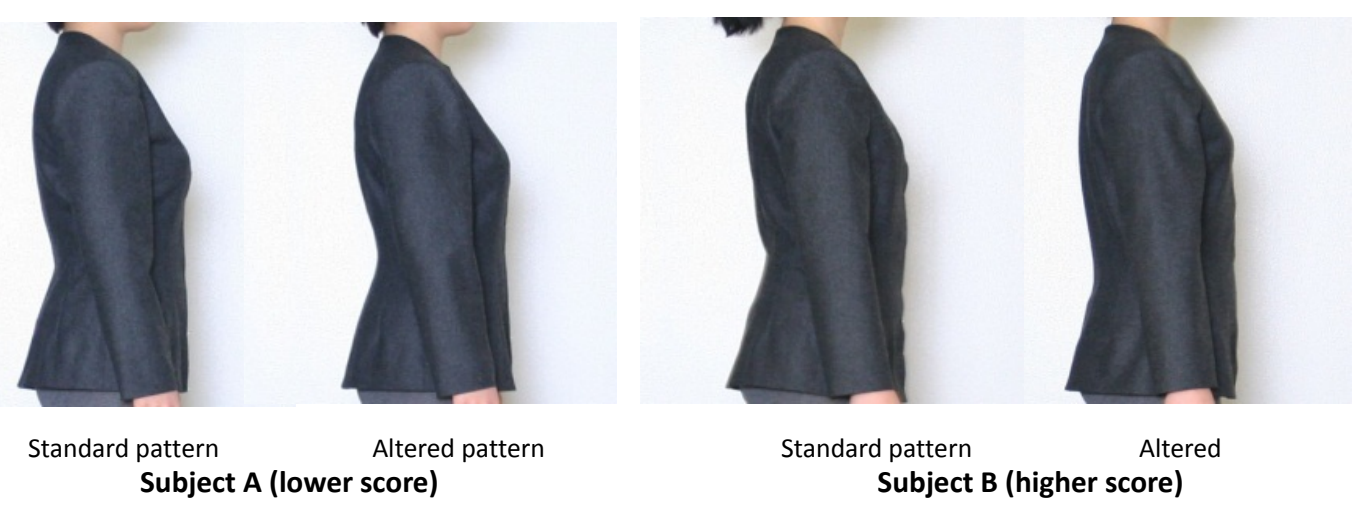

Fig. 9. Comparison of fitting between a suit jacket with standard pattern and a suit jacket with altered pattern for the subjects. 
Pictures of the fitting are shown in Fig. 9. When Subject A in the Low Score group wore the standard jacket, the jacket pulled tight in the upper back area and diagonal wrinkles were formed between the neck and the bottom of the armhole. The hem line was pulled up at the center back. In the altered jacket, the wrinkles were improved and the hem line became straight. The subject mentioned that the standard jacket was tight at the upper back, but the altered jacket was very comfortable. When Subject $B$ in the High Score group wore the standard jacket, the jacket sagged at the lumbar vertebra area and horizontal ripples formed across the upper back. The sag and ripples were improved in the altered jacket, although most of the grade points moved a distance of less than $1 \mathrm{~cm}$.

These results suggest that it may be possible to use body type grade in practice, although more examination and discussion are needed. First, improvements of the fit should be proven scientifically by pressure or sensory evaluation methods. Evaluation of the fit in individuals with lower or higher scores than the present subjects must be done, to consider if the distance is appropriate or not. A higher degree of alterations, which means that the grade points moved larger distances, might be needed. In the previous study, similar results for the factor of forward or posterior shoulder joint were obtained. In the future, the other factors will be examined and the grade rule for body type grade will be established.

\section{References}

1. Lee, S., Kunz, I.G., Fiore, M.A.,Campbell, J.R. (2002): "Acceptance of Mass Customizeation of Apparel: Merchandising Issues Associated With Preference for Product, Process, and Place", Clothing and Textile Researdh Journal, Vol.20, No3, pp138-146

2. Shimosaka, C. snd Matsuyama, Y. (2007):" A Research of Mass Customization of Apparel for Consumer Satisfaction" Otsuma Women's University bulletin. Home economics, Vol. 43, pp.105-106.

3. Ashdown, P. S. and Dunne, L. (2006):"A study of Automated Custom fit: Readiness of the technology for the apparel industry", Clothing and Textile Researdh Journal, Vol.24, No2, pp.121-136

4. Mochimaru, M. and Kouchi M.:" Statistics for 3D human body forms", SAE Digital Human Modeling for Design and Engineering International Conference and Exposition, 2000.

5. M. Mochimaru and M. Kouchi (2009): "Statistics of 3-D body shapes using PCA or MDS and their applications", 17th World Congress on Ergonomics (IEA 2009)

6. Ono, K.(2004):"Pattern Making", Bunka Publishing Bureau, Tokyo, ISBN 4-579-10714-4

7. Watanabe, K.,(2008):"Three Dimensional Body Measurements and CAD Pattern Making", Abstract book- Research paper of International Federation for Home Economics XXI. World Congress, Lucerne, Swiss, pp.22 